\title{
Reduzieren hohe Vitamin-D-Spiegel die Krankheitsaktivität?
}

Fragestellung: Besteht ein Zusammenhang zwischen den Vitamin-D-Spiegeln und der Erkrankungsaktivität bei der schubförmig verlaufenden Multiplen Sklerose (MS) und lassen sich diese Aktivitätsunterschiede auch kernspintomografisch belegen?

Hintergrund: Die Diskussion zur Bedeutung des Vitamin-DSpiegels für die Erkrankungsaktivität der schubförmig verlaufenden MS wird erneut kontrovers geführt, da mehrere Studien Hinweise darauf gegeben haben, dass hohe Vitamin-D-Spiegel mit einer niedrigen Erkrankungsaktivität assoziiert sind und umgekehrt niedrige Vitamin-D-Spiegel mit einer hohen Erkrankungsaktivität korrelieren. Größere Studien über längere Zeiträume liegen dazu nicht vor. Unklar ist ferner, inwieweit Vitamin-D-Spiegel die Wirkung der immunmodulatorischen Therapien, insbesondere der Interferon-Behandlung beeinflussen.

Patienten und Methodik: In die hier vorliegende Studie norwegischer Wissenschaftler wurden 88 Patienten mit schubförmig verlaufender MS aufgenommen. Es wurden die

Løken-Amsrud KI, Holmøy T, Bakke SJ et al. Vitamin D and disease activity in multiple sclerosis before and during interferon- $\beta$ treatment. Neurology 2012; 79: 267-73 Spiegel von 25-HydroxyVitamin-D (25-OH-D) sechs Monate vor Beginn einer Interferon-beta-Behandlung sowie prospektiv für weitere 18 Monate gemessen. Während der 18 Monate andau- ernden Behandlungsphase mit Interferon wurden darüber hinaus fünf kernspintomografische Verlaufskontrollen durchgeführt.

Ergebnisse: Vor der Aufnahme der Interferon-beta-Behandlung war ein Anstieg des 25-OH-D-Spiegels von jeweils $10 \mathrm{nmol} / \mathrm{l}$ mit einer jeweils 12,7\%igen Reduktion für neue T1-Gadolinium-aufnehmende Herde assoziiert $(\mathrm{p}=0,037)$. Darüber hinaus betrug die Reduktion für neue T2-Läsionen 11,7\% $(\mathrm{p}=0,044)$.

Patienten mit einer verstärkten Fluktuation des 25-OH-DSpiegels zeigten eine höhere Proportion von kernspintomografisch nachgewiesenen T1-Gadolinium-aufnehmenden Herden ( $51 \%$ vs. $23 \%, \mathrm{p}=0,004$ ) und einen Trend für neue T2-Läsionen ( $49 \%$ vs. $28 \%, \mathrm{p}=0,052$ ).

Ein Zusammenhang zwischen den 25-OH-D-Spiegeln und der Erkrankungsaktivität war nach der Initiierung der Interferon-beta-Behandlung nicht mehr detektierbar. Darüber hinaus waren die Ergebnisse unabhängig von dem HLADRB1.15-Status der Patienten.

Schlussfolgerungen: Bei unbehandelten Patienten mit schubförmig verlaufender MS sind ansteigende Spiegel von Vitamin D (25-OH-D) mit einer reduzierten radiologisch messbaren Erkrankungsaktivität assoziiert. Dieser Zusammenhang ist unabhängig von dem jeweiligen HLA-DRB1.15-Status der Patienten.

\section{- Kommentar von Volker Limmroth, Köln}

\section{Vitamin D und Multiple Sklerose: Es wird kompliziert}

Dies ist nun noch eine weitere Studie, die eine klare Assoziation zwischen hohen Vitamin-D-Spiegeln und einer niedrigeren Aktivität der Krankheit und umgekehrt, niedrigen Spiegeln und erhöhter Krankheitsaktivität aufzeigt. In der hier vorliegenden Studie wurden kernspintomografische Untersuchungen zur Evaluation und Quantifizierung benutzt. Auch diese Studie ist mit 88 Patienten noch relativ klein, allerdings prospektiv und vom Studiendesign nicht zu kritisieren, auch wenn es etwas überrascht, dass kernspintomografische Unterschiede bereits innerhalb einer Zeitspanne von nur sechs Monaten erkennbar und statistisch signifikant unterschiedlich sind.

Interessant ist aber auch das Ergebnis, dass bei initiierter Interferon-Therapie diese Unterschiede offensichtlich kein signifikantes Niveau mehr erreichen. Dies deutet darauf hin, dass zwar messbare Unterschiede bestehen, diese aber unterhalb der Größenordnungen vom therapeutischen Effekt der Interferon-Präparate liegen. Interessant bleibt auch die Er- kenntnis, dass die genetische Disposition für Autoimmunerkrankungen mit klassischen HLA-Typen diese Ergebnisse nicht beeinflusst hat.

Zusammenfassend gibt uns diese Studie ein weiteres Mosaikbausteinchen zur Klärung der Frage, inwieweit Vitamin D die Erkrankungsaktivität tatsächlich beeinflusst. Damit sammeln beziehungsweise häufen sich die Studien, die eine Assoziation belegen. Der reduzierte Effekt während der InterferonTherapie deutet aber auch darauf hin, dass die Größenordnung dieses möglichen Effekts - sofern er besteht - deutlich unterhalb der therapeutischen Effekte einer Interferon-Behandlung liegt. Damit könnte sich als wichtige Botschaft andeuten, dass Vitamin-D-Spiegel tatsächlich in geringem Umfang die Erkrankungsaktivität beeinflussen und im Zweifelsfall gemessen und angehoben werden sollten. Nichtsdestotrotz brauchen wir dringender denn je ein oder zwei große Studien mit einer ausreichenden Patientenzahl, die diese Fragestellung prospektiv untersuchen und abschließend klären. 